\title{
Do self reported safety behaviours predict childhood unintentional injuries?
}

\author{
R Hapgood, D Kendrick, P Marsh
}

\begin{abstract}
Objective-The aim of this study was to investigate the validity of self reported safety behaviours as a proxy for injuries in unintentional injuries research.

Setting-The study population comprised parents and guardians of children aged 3-12 months in 18 general practices throughout Nottingham $(n=764)$ who responded to a questionnaire on safety practices.

Method-Injury data were collected by searching the primary and secondary care records of each child in the study. Safety behaviour was measured by computing a safety practices score from self reported safety practices for each respondent to a postal baseline questionnaire survey of safety behaviours. The score was used to classify families into low, medium, and high risk of injury occurrence. Two further scores were calculated, firstly for those safety practices which required obtaining an item of safety equipment, and secondly those safety practices requiring behavioural change without cost implications.
\end{abstract}

Results-High risk families were no more likely than low risk families to sustain an injury (odds ratio (OR) 1.08 ; 95\% confidence interval 0.65 to 1.79 ). Medium risk families were also no more likely than low risk families to have a medically attended injury (OR $1.09 ; 0.73$ to 1.61 ) suggesting no association between safety score and future medically attended injury. Similarly, compared to low risk families, medium risk (OR $0.93 ; 0.33$ to 2.61 ) and high risk (OR $0.46 ; 0.08$ to 2.43 ) families were no more likely to have a child admitted to hospital with an injury. There was no correlation between the total number of injuries sustained during the study period and the baseline safety practices score (Spearman's rho $=0.004 ; p=0.917)$. Subgroup analyses for safety behaviours requiring passive and active safety measures did not reveal significant associations with injury outcomes.

Conclusion-Self reported safety behaviours do not appear to be good predictors of childhood unintentional injuries. Further research is required to ascertain valid proxy outcome measures for injury research.

(Injury Prevention 2001;7:14-17)

Keywords: proxy measures; safety behaviours
Accident and emergency services in the UK dealt with an estimated 565000 attendances by children under 5 years during 1996 after unintentional injury. ${ }^{1}$ Unintentional injury is the leading cause of death in children greater than 1 year of age. ${ }^{2}$ Clearly the development and assessment of childhood unintentional injury prevention programmes is a priority for the research community. Such programmes often use intermediate outcome measures and proxies. The widespread use of safety behaviours as a proxy for childhood injuries in injury prevention research would be concerning if they were not strongly associated with injury outcomes.

In injury research, few studies have attempted to validate self reported home safety practices with observations of actual behaviour. One small trial in the UK found a high degree of consistency between self reported practices and observed practices, ${ }^{3}$ but this finding needs confirmation from larger studies. No study has investigated the relationship between self reported home safety practices and eventual injury occurrence in the home.

The aim of this study is to investigate the validity of self reported safety behaviours as a proxy for injuries sustained in the home in unintentional injuries research. In studies of home injury prevention, injuries, and resulting hospitalisation, disability, and death, are usually the outcomes of primary interest. However, because home injuries are relatively rare in the general population, most studies examine effects on proxy outcomes such as safety practices, which are more easily measured. ${ }^{3-10}$ There is therefore a lack of research into self reported safety behaviours as a proxy for injuries in the home.

This study addresses the gap in the literature. Self reported behaviours regarding the supervision of infants and the use of pillows, duvets, toys, baby walkers, hot water, cleaning materials, smoke alarms, stairgates, and fireguards were assessed in a cohort of parents from Nottingham. This was correlated with medically attended unintentional injury of those infants documented in primary or secondary care records.

\section{Methods}

The study population comprised parents and guardians of children aged 3-12 months in 18 general practices throughout Nottingham $(n=764)$ responding to a postal questionnaire that assessed safety behaviours for the families. The questionnaire comprised the baseline data collection for the control group in a cluster randomised controlled trial of injury prevention in primary care. ${ }^{8}$ The response rate to the 
Table 1 Definition of low, medium, and high risk families from the safety scores

\begin{tabular}{llll}
\hline Score $($ range $; n)$ & $\begin{array}{l}\text { Low risk families } \\
\text { score range }(n)\end{array}$ & Medium risk families score range $(n)$ & $\begin{array}{l}\text { High risk families } \\
\text { score range }(n)\end{array}$ \\
\hline Safety practices $(0.00-0.57 ; \mathrm{n}=764)$ & $0.00-0.13(151)$ & $0.14-0.28(485)$ & $0.29-0.57(128)$ \\
Safety equipment score $(0.00-1.00 ; \mathrm{n}=764) \dagger$ & $0.00(476)$ & Not applicable as dichotomised & $>0.00(288)$ \\
Safety behaviours score $(0.00-0.64 ; \mathrm{n}=764) \ddagger$ & $0.00-0.17(209)$ & $0.18-0.26(220)$ & $0.27-0.64(335)$ \\
\hline
\end{tabular}

*All scores were calculated by adding the number of practices categorised as unsafe and dividing by the total number of questions completed regarding safety practices for each respondent. Behaviour was categorised as unsafe if the respondent always or sometimes carried out the unsafe behaviour.

†The safety equipment score was calculated from responses to questions enquiring after those practices involving pieces of safety equipment.

¥The safety behaviours score was calculated from responses to questions enquiring after those behaviours not involving pieces of safety equipment.

questionnaire was $75 \%$. Questions on safety practices in the home were devised to include those injury mechanisms associated with the most frequent ${ }^{1}$ and the most severe ${ }^{2}$ home injuries. The questions on safety practices have previously been found to have acceptable reliability on a test/re-test procedure with $\kappa$ coefficients between 0.70 to $1.00 .^{8}$

The postal questionnaire was mailed with a covering letter from the general practitioner in July 1995. A postcard reminder was sent to non-responders two weeks later and a second questionnaire mailed two weeks after the postcard, if a response had not been elicited. Health visitors identified families on their caseload who they considered may require help completing the questionnaire, and interpreters from the local community health trust contacted those families to carry out structured interviews. Injury data were collected from 1 September 1995 to 30 September 1997 by searching the primary and secondary care records of each child in the study.

Safety behaviour was measured by computing a safety practices score, which was calculated by adding the number of practices categorised as unsafe and dividing by the total number of questions completed regarding safety practices for each respondent. Behaviour was categorised as unsafe if the respondent always or sometimes carried out the unsafe behaviour. This process was repeated for two subgroup analyses, firstly for those safety practices which required obtaining an item of safety equipment, and secondly for those safety practices requiring behavioural change without cost implications. Distributions were examined. Those for the safety practices score and the

Table 2 Frequency of sociodemographic characteristics and history of previous medically attended injury (\%)

\begin{tabular}{lc}
\hline Sociodemographic characteristics and history of previous injury & $\begin{array}{l}\text { Frequency in study population (\%) } \\
(n=764)\end{array}$ \\
\hline Male child & $400(52.6)$ \\
Four or more children & $61(8.2)$ \\
Ethnic group non-white & $50(6.7)$ \\
Lone parent & $98(13.1)$ \\
Unemployed & $76(10.6)$ \\
Non-owner occupation & $284(38.0)$ \\
Overcrowded† & $77(10.3)$ \\
No access to car & $162(21.7)$ \\
Receiving means tested benefitsł & $259(34.9)$ \\
Previously medically attended unintentional injury & $48(6.4)$ \\
Teenage mother & $124(17.3)$ \\
Residing in a deprived area $\$ & $114(14.9)$
\end{tabular}

*One or both parents unemployed.

$\dagger$ Defined as more than one person per room excluding bathrooms and kitchens less than $2 \mathrm{~m}$ wide. $\ddagger$ Defined as receiving a financial benefit from the UK government for which eligibility is based upon income.

$§$ Defined as living in a ward with a Jarman score greater than 30 . The Jarman score is a measure

of general practitioner workload that has been widely used as a proxy measure for deprivation. ${ }^{18}$ safety behaviours score appeared trimodal and were therefore divided into low risk, medium risk, and high risk (see table 1). The safety equipment score was positively skewed and so was divided into those with all items of safety equipment surveyed (low risk), and those with less (high risk). The scores were compared with the following outcome measures: (A) any medically attended unintentional injury, (B) unintentional injury requiring hospitalisation, and (C) number of medically attended unintentional injuries during the study period. The data were analysed using SPSS version 8.0. ${ }^{11} \mathrm{~A}$ sample size calculation indicated that the study had $90 \%$ power at the $5 \%$ significance level to detect a relative risk of any injury occurring in a high risk family when compared to a low risk family of 1.7 (equivalent to detecting a correlation coefficient of 0.3 ), or a relative risk of 1.6 when comparing high to medium risk families.

\section{Results}

Information on safety behaviour at baseline and injury outcomes over the two year study period were available on 764 families. The sociodemographic characteristics are shown in table 2 , and frequencies of unsafe behaviours in table 3.

High risk families were no more likely than low risk families to sustain an injury (odds ratio (OR) 1.08; 95\% confidence interval (CI) 0.65 to 1.79 ). Medium risk families were also no more likely than low risk families to sustain an injury (OR $1.09 ; 0.73$ to 1.61 ) suggesting no association between safety practices score and future medically attended injury. Similarly, compared to low risk families, medium risk (OR $0.93 ; 0.33$ to 2.61 ) and high risk (OR 0.46 ; 0.08 to 2.43 ) families are no more likely to have a child admitted to hospital with an injury. There was no correlation between the total number of injuries sustained during the study period and the baseline safety practices score (Spearman's rho $=0.004 ; \mathrm{p}=0.917$ ), the safety equipment score (Spearman's rho = $-0.003 ; \mathrm{p}=0.939)$ or the safety behaviour score (Spearman's rho $=0.028 ; \mathrm{p}=0.436$ ).

A repeat of the analysis using safety scores derived from those safety practices which required obtaining an item of safety equipment, and secondly those safety practices requiring behavioural change without cost implications, made no difference to the conclusions drawn from the results of the logistic regression (table 4). 
Table 3 Frequency of unsafe practices for childhood unintentional injury (\%)

\begin{tabular}{lc}
\hline Unsafe practice & Frequency (\%) (n=764) \\
\hline Safety behaviour & $107(14)$ \\
Uses a pillow in the cot & $351(45.9)$ \\
Uses a duvet in the cot & $203(26.6)$ \\
Hot water temperature above $54^{\circ} \mathrm{C}$, or does not know hot water temperature and does not use cold & $195(25.5)$ \\
water first in bath & $23(3.0)$ \\
Doesn't check toys for small parts & $113(14.8)$ \\
Possess toys that fit in mouth & $6(0.8)$ \\
Infant left unattended on high surfaces & $128(16.8)$ \\
Infant left unattended in bath & $408(53.4)$ \\
Cord used to tether dummy to child's clothing & $274(35.9)$ \\
Uses baby walker & $37(4.8)$ \\
Drinks hot drinks when holding infant & $165(21.6)$ \\
Does not store cleaning materials out of reach of infant & $76(9.9)$ \\
Safety equipment & $137(17.9)$ \\
Does not have fitted and working smoke alarms & \\
Does not have fitted stairgates & \\
Does not have fitted fireguards &
\end{tabular}

${ }^{\star}$ Excludes families who do not have stairs or fires in the home.

Table 4 Logistic regression analysis for medically attended unintentional injuries and safety behaviour scores

\begin{tabular}{ll}
\hline Logistic regression dependent variable & Odds ratio (95\% confidence interval; $p$ ) \\
\hline Total safety score & \\
$\quad$ Any medically attended unintentional injury & $1.09(0.73$ to $1.61 ; 0.670)$ \\
$\quad$ Medium $v$ low risk & $1.08(0.65$ to $1.79 ; 0.763)$ \\
$\quad$ High $v$ low risk & $0.93(0.33$ to $2.61 ; 0.893)$ \\
Admission to hospital with a medically attended injury & $0.46(0.08$ to $2.43 ; 0.363)$ \\
$\quad$ Medium $v$ low risk & $1.14(0.83$ to $1.55 ; 0.414)$ \\
$\quad$ High $v$ low risk & $2.03(0.86$ to $4.75 ; 0.104)$ \\
Safety equipment score & \\
$\quad$ Any medically attended unintentional injury (high $v$ low risk) & \\
Admission to hospital with a medically attended injury (high $v$ low risk) & $1.30(0.87$ to $1.95 ; 0.203)$ \\
Safety behaviour score & $1.09(0.75$ to $1.59 ; 0.651)$ \\
$\quad$ Any medically attended unintentional injury & $0.80(0.26$ to $2.41 ; 0.688)$ \\
$\quad$ Medium $v$ low risk & $0.76(0.29$ to $2.12 ; 0.624)$ \\
$\quad$ High $v$ low risk &
\end{tabular}

\section{Discussion}

Families reporting more unsafe practices do not seem to be more likely to suffer an unintentional injury to a child than are those reporting safer practices, nor are they more likely to have a child admitted to hospital with an unintentional injury. Moreover, unsafe practices at baseline are not associated with number of injuries sustained during the study period.

This observational cohort study is the first of its kind to our knowledge. There are a number of possible explanations for our study not finding an independent association between safety practices and unintentional injury. Insufficient power is unlikely to explain our results as the sample size calculation indicated the study had $90 \%$ power to detect a relative risk of injury of at least 1.6. If an association does exist between safety practices and unintentional injuries, if it is weaker than this, the use of safety practices as an intermediate outcome measure for injuries would be severely limited. The first possible explanation for our findings is that there may be no relationship, or only a very weak relationship between safety behaviour and subsequent injury, with other factors determining the occurrence of accidents. For example if a family sustains its safe behaviour for the majority of the time, but lapses for a minority of the time, an injury may still result despite the family reporting itself as safe. If this were the case, one might expect there to be a closer association between the use of passive safety measures, such as those items of equipment used to generate our safety equipment score which operate even when family members' safety lapses, and prevention of injuries than with active safety behaviours such as those used to generate our safety behaviours score. However, neither score was significantly associated with unintentional injury occurrence in this study.

Secondly, and importantly, self reported safety practices may not be a valid measure of true safety behaviour. While the questionnaire used in this study has previously been demonstrated to have adequate reliability, ${ }^{8}$ parents may still over report safe practices and under report risky behaviours. ${ }^{12}{ }^{13}$ If this is the case, this could explain the lack of an association between self reported practices and injury. It is also possible that the validity of self reported safety practices varies with the prevalence of the unsafe practice. This phenomenon has been observed with seat belt use. Studies conducted in 1987 and 1988 found that self reports of safety belt use were 21.5 to 27 percentage points higher than observed use, probably because of a social desirability bias in the responses. ${ }^{14}{ }^{15}$ Observed safety belt use subsequently increased over the ensuing 10 years. ${ }^{16} \mathrm{Re}$-examination of the validity of self reported data revealed that self reported estimates were only 2 to 5 percentage points higher than observed estimates. ${ }^{17}$ It is therefore possible that as safety practices become more common, the validity of self reported practices will increase.

Thirdly, inquiring about safety practices may alter behaviour such that individuals become 
safer. If the effect of inquiry on safety behaviours is different across the spectrum of safety behaviours, for example if unsafe families became safer as a result of filling in a safety questionnaire, then this would distort the relationship between safety practices and injury.

The implications of this study are that self reported safety practices, despite being commonly used in injury research, may not currently be good proxy measures in home injury prevention trials. Future research should attempt to validate self reported safety practices as a measure of true behaviour, and to determine the degree of association between validated measures and injury outcomes. In addition, if the prevalence of safe behaviours in the home increases over time, it is possible that the validity of self reported measures will also increase. A validated proxy measure such as self reported safety practices is desirable if injury prevention trials are to be conducted in a cost effective and pragmatic manner.

1 Department of Trade and Industry. Home accident surveillance system. 20th Annual report. London: DTI, 1998.

2 Office for National Statistics. Mortality statistics: injury and poisoning 1996. England and Wales. London: ONS, 1998.

3 Clamp M, Kendrick D. A randomised controlled trial of general practitioner safety advice for families with children under 5 years. BMF 1998;316:1576-9.
4 Dershewitz RA, Williamson JW. Prevention of childhood household injuries: a controlled clinical trial. Am f Public Health 1977;67:1148-53.

5 Jenkins HML, Blank V, Miller K, et al. A randomised singleblind evaluation of a discharge teaching book for pediatric patients with burns. F Burn Care Rehabil 1996;17:49-61.

6 Katcher ML, Landry GL, Shapiro MM. Liquid-crystal thermometer use in pediatric office counselling about tap water burn prevention. Pediatrics 1989;83:766-71.

7 Kelly B, Sein C, McCarthy PL. Safety education in a pediatric primary care setting. Pediatrics 1987;79:818-24.

8 Kendrick D, Marsh P, Fielding K, et al. Preventing injuries in children: cluster randomised controlled trial in primary care. BMF 1999;318:980-3.

9 Thomas KA, Hassanein RS, Christopherson ER. Evaluation of group well-child care for improving burn prevention of group well-child care for improving burn p.
practices in the home. Pediatrics 1984;74:879-82.

10 Woolf A, Lewander W, Filippone G, et al. Prevention of childhood poisoning: efficacy of an educational program carried out in an emergency clinic. Pediatrics 1987;80:35963.

11 SPSS Inc. SPSS for Windows version 8.0. Chicago, IL: SPSS Inc, 1998.

12 Scott I. You can't believe all that you're told: the issue of unvalidated questionnaires. Inj Prev 1997;3:5-6.

13 Mickalide A. Threats to measurement validity in selfreported data can be overcome. Inj Prev 1997;3:7-8.

14 Robertson LS. The validity of self-reported behavioural risk factors: seatbelt and alcohol use. F Trauma 1992;32:58-9.

15 Centers for Disease Control. Comparison of observed and self-reported seat belt use rates-United States. MMWR Morb Mortal Wkly Rep 1988;37:549-51.

16 McGinnis J, Lee P. Healthy people 2000 at mid decade. fAMA 1995;273:1123-9.

17 Nelson D. Validity of self reported data on injury prevention behavior: lessons from observational and self reported surveys of safety belt use in the US. Inj Prev 1996;2:67-9.

18 Jarman B. Identification of underprivileged areas. BMF $1983 ; 286: 1705-9$ 